\title{
Una nota sobre tasa de interés y eficiencia
}

\author{
Joaquín Arriola Palomares*
}

Es bastante usual el uso de la expresión "tasas de interés negativas", en referencia al hecho de que las tasas activas y pasivas (i) sean inferlores a la inflación medida (p):

$$
i-p<0
$$

se afirma que cuando esto ocurre, la asignación del crédito es "ineficlente".

Este tipo de valoraciones responde a una teorización muy deficiente sobre el carácter de la moneda, de modo que las expresiones entrecomilladas tienen una muy escasa capacidad analítica, un carácter teórico dudoso y nos dan una interpretación sesgada de la realidad. ¿La fórmula anterior significa realmente la existencia de tasas de interés negaívas? SI el incremento de la inflación medida es superior a las tasas de interés, ¿debemos concluir que la asignación de los recursos crediticios será ineficiente, o quizá menos eficiente que con tasas de interés superiores a la inflación medida? ¿Es la relación entre el indice de precios y la tasa de interés un indicador de eficiencia?

La respuesta es negativa en los tres casos, y esto por tres razones principales: en primer lugar, el dinero carece de valor de cambio, en segundo lugar, efecto macroeconómico. la actividad de intermediación

- Agradezco a Aquiles Montoya, Carlos Briones y a Alvaro Trigueros Arguello sus útiles comentarios, no siempre bien aprovechados. 
financiera es algo más que mediadora en el proceso de acumulación, teniendo un carácter predador sobre el mismo. Tercero, a nivel microeconómico, el coste de oportunidad de un crédito no se puede medir con el indicador de la inflación. Veamos estas afirmaciones con un poco más de detalle.

1. El dinero no es sino una forma alienada de la mercancia: carece de existencia autónoma, al margen de la producción circulación de mercancias. Su caracter de equivalente general dota al dinero de valor simbólico, pero no de valor real, que sólo lo tienen los productos del trabajo humano. El dinero no tiene valor de cambio, el cual es específico de los productos del trabajo humano producidos como mercancias, es decir, valores de uso destinados a ser intercambiados. El "poder de compra" de la moneda no es el valor de la moneda en un momento dado. El "valor de la moneda" es una expresión que carece de sentido, puesto que la moneda carece de medida: en tanto que reflejada en la relación entre todas las mercancias, y reflejo de cada una de ellas de forma diferenciada, la moneda misma es la medida-lo indiferenciado:

"El poder de compra de la moneda expresa la dimensión tempora. de la circulación cuando toma la forma D-M-D. Este poder de compra remite la cantidad de un objeto que puede ser comprado con un monto dado de moneda en una cierta fecha a la cantidad del mismo objeto que puede ser comprada con el mismo monto de moneda en otra fecha. Pero el objeto o conjunto de objetos es arbitrario ya que la moneda puede comprar todo. Por consiguiente, la ambivalencia de la moneda se expresa por el caracter irremediablemente convencional de toda medida de su poder de compra".'

Desde el momento en que el valor de uso del dinero es facilitar el acceso a la ganancia, su nivel esta limitado por la tasa de ganancia media esperada. ${ }^{2}$ El pago de intereses no es más que una participación en el excedente generado en la producción de mercancias, una extracción que limita la capacidad de realización productiva de la plusvalia, siendo en la misma medida un freno a la acumulación del capital. Pero en ningún sentido se puede decir que la tasa de interés sea el "precio" del dinero, salvo en la lógica ateórica del marginalismo. No hay un valor de cambio para el dinero como tal, por tanto no existe nada parecido a una tasa de interés "natural" en torno a la cual deba fluctuar la tasa de mercado. La existencia de tasas de interés inferiores al indice de inflación, es decir inferiores a la evolución de la capacidad adquisitiva del dinero (10 que se denomina tasas de interés "negativas") no significa ninguna referencia a un supuesto desequilibrio: es un absurdo teórico la hipótesis de que las tasas de interés "naturales" deban ser "positivas"." 
La existencia de tasas de interés activa inferiores a la evolución de la inflación medida es una forma de apoyo al proceso de acumulación tan válida como cualquier otra, sujeta por supuesto al buen uso de la misma: definición de un horizonte temporal de graduación que contemple la reducción de la diferencia entre $p$ mayor que i activas, y selectividad en el uso de la medida en coherencia con un plan de desarrollo. A largo plazo, nosotros abogariamos por una optimización en el uso de los recursos que considerase la minimización de las tasas activas, reales sujeta a las tasas pasivas necesarias para alcanzar el nivel de depósitos requerido para mantener la tasa de acumulación planificada. La remuneración de los depósitos y el coste del crédito (diferencia entre tasas activas y pasivas) cuando se fijan en orden a maximizar la rentabilidad de la actividad de intermediación, no es socialmente eficiente, pues reduce la tasa de acumulación real en la misma medida en que se desvia el excedente de su uso productivo. Pero pasar la actividad de intermediación financiera de la lógica privada de la rentabilidad a la lógica social de la acumulación, exige el control público sobre la actividad bancaria.

2. Si el dinero carece pues de valor de cambio, la asignación al mismo de un "precio" (i) otorga a este un caracter esencialmente diferente al de los otros precios: su número es fruto de una convención, no de un hipotético equilibrio, inexistente entre la oferta y demanda de dinero. La idea de que una condición necesaria para que la asignación del crédito sea eficiente es que el precio del dinero sea mayor al crecimiento medido de los precios, es un disparate teórico.

En el nivel de la reproducción social o macroeconómica, una tasa de interés mayor que cero siempre es positiva, significa que el uso de dinero recibido como crédito sea en la circulación, sea en la producción, debe transferir una fración del excedente hacia la institución financiera, fracción por el número que designa el precio (convencional) de la moneda.

En este sentido, se puede burlar parcialmente la necesaria transferencia de excedente aumentando los precios de las mercancias que se van a producir o realizar con el dinero obtenido como crédito: asi, la fórmula

$$
P_{1+1} / i_{1}
$$

tiene un sentido diferente si es mayor o menor que uno. Si el cociente se situase entre 1 y 0 , sería un indicador de dimensión del excedente transferido fuera del sector productivo (circulación y/o producción). Por 
el contrario, un valor mayor que 1 del cociente refleja la contradicción entre la función de medio de circulación y de reserva de valor del dinero, la resistencia del capital productivo a ser expulsado de la valorización, su deseo de mantenerse como capital dinetario y capital mercancia, en un proceso de metamorfosis ininterrumpido. Un signo negativo en el cociente por reducción del indice de precios refleja una situación del primer tipo; si el cociente es negativo porque $i<0$, nos encontramos con una situación de desequilibrio en el proceso de valorización único caso en que la asignación del crédito puede no corresponder a una lógica de valorización coherente.

La transferencia de excedente del sector productivo al financiero reduce la velocidad de la acumulación, y la valorización del capital sin producción de mercancias (D-D') se manifiesta introduce una distorsión en la regulación por la moneda: la existencia de tasa de interés positivas es un factor inflacionario siempre que su número sea superior a la velocidad de producción de plusvalía o excedente. La relación

$$
\text { ve }
$$

siendo e la tasa de crecimiento del excedente producido, nos mide el efecto inflacionario de las tasas de interés positivas, mayor cuanto más elevado sea el cociente. Este efecto dejaría de producirse solo cuando el valor del cociente sea inferior a 1.

3. Quien solicita un crédito para realizar una actividad de producción (o una actividad de consumo), no confronta la tasa de interés con el nivel de precios, sino con la rentabilidad de la operación productiva (tasa de ganancia esperada).

Es un error analítico buscar la eficiencia del crédito del lado de quien lo asigna, ya que esta se encuentra del lado del uso. La rentabilidad de la actividad bancaria se encuentra en el diferencial entre las tasas activas y pasivas, pero el nivel de las tasas de interés de ninguna forma es un indicador de eficiencia, ni de lo contrario.

Además, la moneda tampoco se puede definir en términos microeconómicos, pues su propia expresión social implia la relación entre agentes. Se puede establecer una fundamentación microeconómica de la moneda, en este caso, de las tasas de interés, pero la eficiencia del crédito siempre se define en el nivel macroeconómico- las operaciones individuales no definen niveles de eficiencia, sino de rentabilidad.

Por tanto, no es con la evolución del nivel general de precios con lo que hay que confrontar las tasas de interés, sino con la evolución de la 
tasa de ganancia y la renta individual (para simplificar, los salarios), para lograr alguna información relevante sobre la eficiencia de la asignación del crédito de producción y de consumo: la relación significativa seria

$$
\frac{c w+(1-C) G}{i}
$$

siendo w la evolución de los salarios nominales, G la evolución de las ganancias, i la tasa de interés activa nominal, c la proporción de créditos de consumo familiar y (1-c) la de créditos de producción o de inversión (incluido el consumo de las empresas).

Poniendo un ejemplo práctico, entre 1985 y 1987 se dieron en El Salvador las tasas de interés supuestamente más negativas: las tasas de activas "reales" habrian sido $-8.2^{4}$, luego habrian sido los anos de máxima ineficiencia del sistema crediticio.

Ya hemos indicado que desde el punto de vista de la eficiencia en el uso del crédito, las tasas reales son las nominales, y no las deflactadas. Además, aplicando la fórmula (4) , obtenemos la siguiente relación de eficiencia del crédito para la economia salvadorefa en los anos 1985 a 1987:

$$
\frac{c 27.1+(1-c) 36.8}{16}
$$

Desconocemos el valor de c. Para ilustrar el ejemplo, a partir de la distribución de la cartera de créditos por garantia, plazos, sector económico y usuario 6 , supongamos con cierta laxitud que los créditos de consumo familar representaron la tercera parte del total; en ese caso, el cociente seria:

$$
\frac{33.2}{16}=2.1
$$

que en todo caso es de signo y sentido inverso al que se deduce de la denominada "tasa real" (la fórmula (2) tiene un valor para esos anos de 1.6; que sea inferior al cociente de (4) 10 podemos entender como resultado de la existencia de un efecto de crecimiento productivo positivo en términos reales). Lo que nos esta diciendo el cociente anterior, es que la asignación del crédito facilitó la dinámica de la acumulación. Po- 
driamos construir una serie temporal que nos reflejase la evolución de la eficiencia relativa de la asignación del crédito a partir de la fómula (4). En todo caso, solo cabria hablar de ineficiencia en aquellas situaciones en que dicho cociente fuera inferior a 1, lo cual no se ha dado nunca en todo el periodo de banca nacionalizada. Podriamos por tanto hablar de eficiencia relativa de la asignación y uso del crédito con la banca nacionalizada, nunca de ineficiencia.

En otro orden, habria que cuestionarse quien es más "ineficiente": el sistema bancario público, incapaz de recuperar la mora, o los capitalista privados, incapaces de pagar sus deudas. La misma "Iógica" con la cual justifica el gobierno la incautación al sector público de la banca, se podria aplicar para llevar a cabo la socialización de los capitales privados morosos.

Sin entrar en mayores detalles, creemos que de lo anterior se deriva con facilidad que el supuesto debate técnico que se da en nuestro país en torno al sistema bancario entre el BCR, Cenitec, Fusades y otros organismos de "pensamiento" e intervención económico es un debate falso: es una discusión técnica sin teoria y por lo tanto dicha discusión solo sirve para sustraer a la opinión pública la fundamental dimensión política de las medidas que el gobierno esta imponiendo en el funcionamiento y orientación del sector financiero.

\begin{tabular}{|lccc|}
\hline & \multicolumn{4}{c|}{$1985-1987$} & \\
\hline & $\begin{array}{c}\text { variación de } \\
\text { salarios }\end{array}$ & $\begin{array}{c}\text { \% sobre el total } \\
\text { de salarios }\end{array}$ & $\begin{array}{c}\text { variación del } \\
\text { valor agregado }\end{array}$ \\
\hline Manufacturas & 26.9 & 37.37 & 72.4 \\
Construcción & 18.8 & 6.94 & 62.6 \\
Comercio & 20.0 & 19.27 & 86.7 \\
Transporte & 45.0 & 4.27 & 73.0 \\
Servicios & 34.7 & 16.95 & 44.8 \\
financieros & 34.0 & \\
\hline
\end{tabular}

(1) Incremento del valor agregado 1985-1987: 63.9\%

(2) Incremento de los salarios no agricolas: $27.1 \%$

(3) Incremento del excedente (1-2) 1985-1987: $36.8 \%$

(4) Tasa de interés activa nominal media 1985-87: 16\%

(5) Tasa de interés activa deflactada media 1985-87: -8.2\% 


\section{Notas}

1. Michel Aglietta y Andre Orlean: La violencia de la moneda Siglo XXI, México 1990 pág. 78.

2. En efecto: la demanda de créditos depende de las expectativas de ganancia de los empresarios. Por tanto, las tasas de interés activas se fijarán alrrededor de la tasa media esperada de ganancia, única referente posible de "equilibrio" para la tasa de interés.

3. K. Cole, J. Cameron y Ch. Edwards: Why economists disagree Longman, Nueva York 1983, pp. 216-217.

4. "La reforma del sistema financiero: es oportuna la reprivatización?" en Política Económica vol. 1 núm. 2 pág. 6, agosto-septiembre 1990.

5. El incremento de los salarios para el período lo obtenemos ponderando de la evolución de los salarios en los sectores de la manufactura, construcción, comercio, transporte y servicios financieros. No incluimos la de los salarios agricolas por su escaso peso relativo y por que serla absurdo suponer que los asalariados agricolas puedan ser sujetos de crédito en este sistema. Las estimaciones de la evolución sectorial de los salarios nos la facilitó Aquiles Montoya, el cual las ha estimado para un trabajo en curso de realización. La evolución de las ganancias la asimilamos a la del valor añadido en los sectores considerados, descotada la participación de los salarios.

6. "Análisis y recomendaciones del SITRABIF para la solución de la actual crisis bancaria en el marco de la negociación " en Coyuntura Económica año 5 núm. 29, marzo-abril 1990. 